\title{
A telehealth system for Parkinson's disease remote monitoring. The PERFORM approach.
}

\author{
Jorge Cancela, Matteo Pastorino, Maria T. Arredondo and Olivia Hurtado
}

\begin{abstract}
This paper summarizes the experience and the lessons learned from the European project PERFORM (A sophisticated multi-parametric system FOR the continuous effective assessment and monitoring of motor status in Parkinson's disease and other neurodegenerative diseases). PERFORM is aimed to provide a telehealth system for the remote monitoring of Parkinson's disease patients (PD) at their homes. This paper explains the global experience with PERFORM. It summarizes the technical performance of the system and the feedback received from the patients in terms of usability and wearability.
\end{abstract}

\section{INTRODUCTION}

PERFORM system targets the remote monitoring and management of patients with Parkinson's Disease (PD). PD is one of the most common neurodegenerative disorders. It occurs in about $1 \%$ of the population over the age of 60 and its prevalence increases with age. Advancements in treatment for chronic diseases have resulted in reduced length of hospital stay, and in some cases, the avoidance of hospital visits. Telemedicine brings healthcare delivery to the home environment by connecting the patient with medical professionals. It is not intended to replace health professional care, but rather to enhance the level of care [1].

The major motor disturbances in PD are bradykinesia (i.e. slowed movement), hypokinesia (small amplitude movements), resting tremor, rigidity, and postural instability. These major motor features of PD are associated with, and are largely a result of, the loss of dopaminergic innervation of the basal ganglia. Although a genetic predisposition has been identified in a subset of patients with PD, several other risk factors for PD have been recognized [1-3]. The cause and etiology of PD are largely unknown [2-6]. PD is typically characterized by severe, unpredictable and abrupt changes in the patient motor performance whereby OFF periods, characterized by the temporary loss of drugs effectiveness, alternate, sometimes within minutes, with ON periods, during which the medication effectively attenuate motion symptoms. The loss of dopaminergic neurons compromises the speed, the automaticity and fluidity of

Manuscript received February 4, 2013. This work is partially funded by the European Commission under the PERFORM project (7th Framework Programme)

J. Cancela is with the CEI Campus Moncloa, UCM-UPM, Madrid, Spain (e-mail: jcancela@1st.tfo.upm.es).

M. Pastorino and M.T. Arredondo are with Life Supporting Technologies, Technical University of Madrid (UPM), Madrid 28040 Spain (e-mail: mpastorino@1st.tfo.upm, mta@1st.tfo.upm.es).

O. Hurtado is with the Departamento de Farmacología, Facultad de Medicina, Universidad Complutense de Madrid, Madrid, Spain (e-mail: olhurtad@med.ucm.es). movements. As the disease evolves, PD patient's motion becomes slower and tremoric and the response to medication fluctuates along the day (ON-OFF periods). In addition, the presence of involuntary movements (dyskinesias) deteriorates voluntary movement in advanced state of the disease.

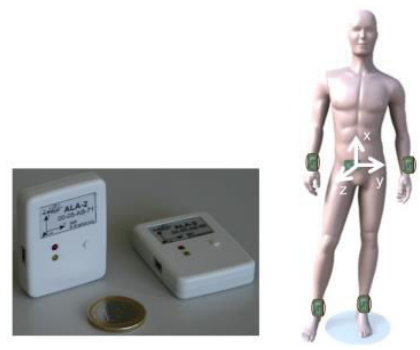

Fig. 1. Sensors used for data collection and their position over the patient's

\section{CURRENT PRACTICE AND PERFORM APPROACH}

\section{A. Current practice}

Currently, motor assessment in PD is mainly based on historical information, home diaries and neurological examination during visits to the clinic. These methods clearly suffer from many drawbacks: data from these sources can be highly subjective, they rely on the patient's memory and perception of his own symptoms and they depend on the physician's experience on the field. Moreover, most of the patients may not be aware of mild symptoms, they may not necessarily understand medical terminology, or they may unconsciously exaggerate or attenuate symptoms severity. Finally, PD can affect short-term memory. In an attempt to solve these problems and to find more objective assessments, several rating scales have been designed and used. Among them, the Unified Parkinson's disease Rating Scale (UPDRS) [7] is the most widely used. This rating scale tries to quantify selected symptoms and parkinsonian signs in a 5 points scoring system (from 0 for no sign, to 4 for a marked severity of the sign). Unfortunately, the UPDRS, like any other semi-objective rating scale, has several limitations. In fact intra and inter-observer inconsistencies and bias by subjectivity issues related to the historical information have been demonstrated. Moreover, the pattern and severity of PD symptoms may vary considerably during the day, while clinical rating scales only provide moment-to-moment assessments; and finally, measurements of motor functions made in the clinic may not accurately reflect the actual functional disability experienced by the patients in their daily life. All those methods described above provide limited information. 


\section{B. PERFORM system}

PERFORM is a telematic platform for PD remote monitoring developed in the last years by a European Consortium of SMEs, large companies, Universities and research centers [8]. The current status of the project is a fully operative prototype which has been tested in three different hospitals across Europe: University of Navarra Medical School Hospital (Spain), the University of Ioannina Hospital (Greece) and the Nuovo Ospedale Civile S.Agostino-Estense of Modena (Italy).

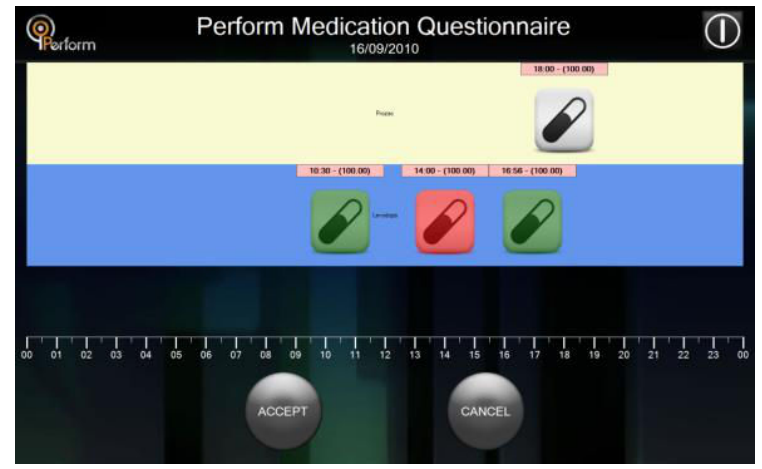

Fig. 2. Medication intake screen. The dose and type of pills is indicated by the appropriate image in order to help the end user remember what kind of medication he/she took and when. By clicking on the prescribed doses the user is able to confirm that the medication was taken at the prescribed time (or declare the real intake time by moving the pill along the horizontal time line). The user can also declare the dosage taken. In order to add a medication intake that was not scheduled, the user can click on the empty space of the desired medicine rectangle.

PERFORM platform was composed by a set of wearable sensors devices for the recording of the motion signals and a set of software algorithms for the signal processing (Fig. 1). The hardware was formed by a set of four tri-axial accelerometers positioned at each patient limb used to record signals from legs and harms; a belt sensor, composed by an accelerometer and a gyroscope, used to record body movement accelerations and angular rate; and a data logger used to receive and store all recorded signals in a SD card. All sensors transmit data using Zigbee protocol to the logger device, with $62.5 \mathrm{~Hz}$ sampling rate before a synchronization phase. All accelerometers transmit data at the same time and no retransmission of lost packets has been implemented in order to save battery. The system is characterized with a global dataloss of $1.24 \pm 0.58 \%$.

Apart from the wearable sensor network, each patient was provided with touch-screen PC at his/her home. The application installed in the PC carries out the signal processing tasks. Once the raw data is downloaded from logger to the PC, it is automatically processed. This software is responsible for the identification and quantification of the patient.A customized graphical user interface (GUI) has been designed to allow the patients enter other useful information. The user could interact with it either with the mouse or with the touch screen modality. Using a touch-screen system offers interesting benefits in the PERFORM case [9]. Touching a visual display of choices requires little thinking and it is a form of direct manipulation easier to learn. Touch screens have easier hand-eye coordination than mice or keyboards and they are the fastest pointing device.

Besides, the GUI was tested on every phase of the pilots and redesigned according to the users' feedback. The software allows the patients to insert the following information:

- $\quad$ medication intake (kind, dose and time) (Fig. 2)

- $\quad$ meals (type of food, amount, time) (Fig. 3)

- PDQ-39. An standard questionnaire for the evaluation of physical, emotional and psychosocial aspects of Quality of Life (QoL) in PD patients [10].

The nature unsupervised environments creates a number of challenging problems with regards to signals interpretation and signals quality validation [11]. Collecting this information is essential in order to create a context and to make the signal processing outcome useful in this sort of environments. In fact, motor behavior strongly depends on the assumption of the medication (in the usual patient's dosage) and the metabolism of the drug is influenced by the diet (proteins or fats).

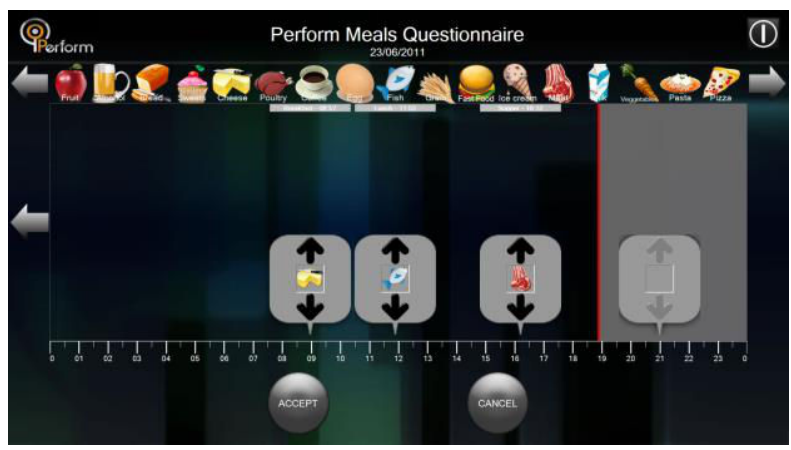

Fig. 3 Meals questionnaire screen. Here the patient is able provide information about the meals taken during the day. The user can compose any of the main meals of the day by dragging selected food products into the appropriate containers representing Breakfast, Lunch, Dinner, and Supper. Each container can be moved along the timeline presented on the bottom of the page. In this way, the patient indicates when the meal was taken (the time is shown at the top of the meal container).

\section{METHODS}

\section{A. Data collection}

PERFORM followed an iterative design process. Three different phases where designed involving 92 PD patients and 20 health subjects. On each phase of the design raw signals were collected using the wearable sensor network.

- Phase I. A preliminary version of the system was tested with 20 healthy subjects. Also this initial phase was used to test the technical performance of the platform in terms of data transmission and communication, identify bugs and redesign the system.

- Phase II. The second phase was carried out in the hospital under the supervision of the doctors. The goal of this phase was to validate not only the technical performance of the system but also the 
clinical compliance of the system. A total number of participants of 36 PD patients were involved.

- Phase IIII: It consisted in the evaluation of final PERFORM prototype. In this last phase the final version of the system was tested in everyday practice at patients' home. The total number of participants was 44 PD patients plus 12 patients with Parkinsonisms (Parkinsonism is any condition that causes a combination of the movement abnormalities seen in Parkinson's disease such as tremor, slow movement or muscle stiffness).

\section{B. Inclusion and exclusion criteria}

Patients fulfilling the following criteria were eligible for the study: Diagnosis of Parkinson's disease, ages of 18-85, ambulatory and capable of complying with study requirements, receiving stable dopaminergic treatment, experiencing motor fluctuations, presence of a responsible caregiver who can cooperate with patient and Perform Team Exclusion Criteria: Dementia, psychosis (simple visual hallucinations excluded), significant systemic disease (such as: cancer, hepatic or kidney dysfunction, etc.), previous Deep Brain Stimulation or Implant of other stimulators

Before to begin the protocol each patient was offered a written description of the study in his/her own language as well as an oral explanation of the research expressed in terms that would have the best chance of being understood. The experimental nature of this study, its inherent risks and discomforts, and its potential for improving the treatment of PD was discussed. Then clinician obtained informed consent and gave a letter with a synopsis of Perform Protocol for family doctor to inform him about main issues of the study.

\section{Data processing}

In order to assess the status of the patient the patient's computer was equipped with ad-hoc software for the detection and quantification of the PD symptoms. The technology used is based on machine learning algorithms; specifically in classificators (knowledge about a given problem is learnt from examples also called the training data). As general approach the raw signal is preprocessed with a linear interpolation algorithm in order to fix the dataloss holes. Then, the signal is split out in "epochs" using a slide window (between 1 and 5 seconds length). For each "epoch" different features are calculated (e.g. rms, entropy or range). These features will feed the classificators which were previously trained using the UPDRS as reference. Data from the Phase II were used to train and validate these algorithms. The window length, classificator type and features that better fit for each symptom were already discussed in detail: tremor [12], [13], levodopa-induced dyskinesias [14], [15], bradykinesia [16], [17] and the assessment of gait [18].

\section{Users'feedback and wearability}

The evaluation of the system wearability was performed with the involvement of a subset of 24 users, 18 males and 6 females from the Phase IV of the PERFORM pilot. Their age ranged between 52 and 76 years old. For the posture analysis, all patients were asked to stand up and stay still for a couple of minutes, while an observer completed the scoring of REBA evaluation based on the posture of trunk, neck, legs and arms [19]. Patients were also asked to read a number of statements and provide a rating between 0 (low) and 20 (high), corresponding to their level of agreement with each statement with regards to emotions, anxieties and harm caused by the system. REBA is a methodology for assessing how wearing a computer affects the user in terms of physiological energy expenditure, the biomechanical effects due to changes in movement patterns, posture and perceptions of localized pain and discomfort due to musculoskeletal loading, and perceptions of well- being through comfort assessment. In order to evaluate the users' experience with the symptom an informal interview was held with the patients in Phase III.

\section{RESULTS}

\section{A. Technical performance}

For each symptom, a dedicated algorithm processes the relevant signals, detects the symptom episode and quantifies it into a severity scale from 0 to 4 , according to the UPDRS scale for PD's patients. About the technical performance of the system it shows an accuracy of $93.73 \%$ for the classification of levodopa induced diskynesias (LID) severity [14], a $86 \%$ of bradykinesia severity [17] and $87 \%$ for tremor [12]. As well as it has developed a special module for the assessment of the gait parameters i.e. step frequency, velocity, arm swing frequency and entropy of the gait signal (entropy is a good measure of the randomness of the signal and it is an excellent discriminator of the On/Off status) [18]. These results were obtained using support vector machines and decision trees (depending on the symptoms).

\section{B. Users feedback}

After the chat with the patients it is possible to conclude that the experience with the touch-screen PC was well accepted; no patient reported problems using it and all of them were able to carry out the monitoring sessions with the instructions provided. Most of the issues reported from the patients were fixed with minor modifications of the system $91.5 \%$. Most of the changes $(61.11 \%)$ where related to a bad implementation of the software i.e. problems with the webcam drivers, microphone drivers and software bugs. The other $38.89 \%$ of the patients complains where related to usability problems e.g. text too small or a not enough intuitive screen.

\section{System wearability}

The majority of the patients did not feel any discomfort/pain at most of their body parts. Only few patients (8 out of 24 patients) felt extremely week discomfort/pain at overall assessment of their body. None of the patients had the perception of any kind of harm (e.g. headache, pain, itching, irritation, etc.) caused by the devices. The interview revealed some emotional and appearance issues of a group of patients (6 out of 24). These 
patients had some concerns about the impression they make to others when wearing the devices. They would feel much more comfortable if the devices were not visible or if they had to wear them only at home. All participants agreed that the provided solution did not obstruct them in everyday activities neither limited their activities in an effective way. The only concern expressed by a number of patients was regarding the proper attachment of the current appliance. The effect of the wearable device to patients was always "low", confirming the wearability of the system.

\section{DISCUSSION}

Telehealth is more than using wearable sensors at patients' home, especially, when we are talking about chronic disease management. It means to involve patients and caregivers in the healthcare path and shift their role from a passive position to an active position. And in consequence, transform them in main actors of the healthcare process.

The first and most obvious reason is that home telehealth systems need the participation and cooperation of patients and caregivers to work efficiently. In the PERFORM context the algorithms developed have achieved a good accuracy. Nevertheless, in order to achieve a good understanding of the disease in the patient and to build a profile of the patient, context information (i.e. food and medication intakes) is required. That means it is needed to invest time and effort in the patient and caregiver training, to capacitate them with the adequate education to use the system properly as well as follow the best practices for GUI design. Moreover, it is important to translate them the importance of the selfassessment and the responsibilities they should take care.

\section{CONCLUSION}

From the technical point of view, PERFORM has shown the feasibility of using wearable accelerometers in combination with machine learning algorithms in order to perform a continuous monitoring of PD patients. Also, patients have shown a good acceptance of the system. As it was discussed in this work, the main challenges for these sorts of systems are the wide range of symptoms, the variability of the symptoms and also the uncertainty working on unsupervised environments. Due to these reasons get the patients and caregivers involvement should be one the first issues to address during the design process.

Next steps must prove the feasibility of the system in a large-scale, long-term and randomized controlled trial in order to show the feasibility of the system for its deployment in the clinical practice.

\section{ACKNOWLEDGMENT}

Authors thank the PERFORM (Grant Agreement 215952) consortium for their contribution to this work, especially the University of Navarra Medical School Hospital, the University of Ioannina Hospital and the Nuovo Ospedale Civile S.Agostino-Estense of Modena.
Research by Jorge Cancela has been partly supported by a PICATA predoctoral fellowship of the Moncloa Campus of International Excellence (UCM-UPM).

\section{REFERENCES}

[1] J. Polisena, D. Coyle, K. Coyle, and S. McGill, "Home telehealth for chronic disease management: a systematic review and an analysis of economic evaluations.," International Journal of Technology Assessment in Health Care, vol. 25, no. 3, pp. 339-349, 2009.

[2] Z. Gan-Or, N. Giladi, U. Rozovski, C. Shifrin, S. Rosner, T. Gurevich, A. Bar-Shira, and A. Orr-Urtreger, "Genotype-phenotype correlations between GBA mutations and Parkinson disease risk and onset.," Neurology, vol. 70, no. 24, pp. 2277-2283, 2008.

[3] A. Orr-Urtreger, C. Shifrin, U. Rozovski, S. Rosner, D. Bercovich, T Gurevich, H. Yagev-More, A. Bar-Shira, and N. Giladi, "The LRRK2 G2019S mutation in Ashkenazi Jews with Parkinson disease: is there a gender effect?," Neurology, vol. 69, no. 16, pp. 1595-1602, 2007.

[4] B. Kandinov, N. Giladi, and A. D. Korczyn, "Smoking and tea consumption delay onset of Parkinson's disease.," Elsevier Ltd, 2009.

[5] B. S. Shastry, "Parkinson disease: etiology, pathogenesis and future of gene therapy.," Neuroscience Research, vol. 41, no. 1, pp. 5-12, 2001.

[6] C. Wider and Z. K. Wszolek, "Etiology and pathophysiology of frontotemporal dementia, Parkinson disease and Alzheimer disease: lessons from genetic studies.," Neurodegenerative Diseases, vol. 5, no. 3 4, pp. 122-125, 2008.

[7] C. Goetz, W. Poewe, O. Rascol, and C. Sampaio, "The Unified Parkinson's Disease Rating Scale (UPDRS): Status and Recommendations," Society, vol. 18, no. 7, pp. $738-750,2003$

[8] "PERFORM project official website," 2012. [Online]. Available: http://www.perform-project.eu/.

[9] N. Caprani, N. E. O'Connor, and C. Gurrin, "Touch screens for the older user," 2012.

[10] P. Martínez-Martín and B. Frades Payo, "Quality of life in Parkinson's disease: validation study of the PDQ-39 Spanish version. The Grupo Centro for Study of Movement Disorders.," Journal of Neurology, vol. 245 Suppl, pp. S34-S38, 1998.

[11] N. H. Lovell, S. J. Redmond, J. Basilakis, T. Shany, and B. G. Celler, "Telehealth technologies for managing chronic disease - experiences from Australia and the UK," Engineering in Medicine and Biology Society EMBC 2010 Annual International Conference of the IEEE, vol. 2010, pp. 5267-5269, 2010

[12] G. Rigas, A. T. Tzallas, M. G. Tsipouras, P. Bougia, E. E. Tripoliti, D. Baga, D. I. Fotiadis, S. G. Tsouli, and S. Konitsiotis, "Assessment of Tremor Activity in the Parkinson's Disease Using a Set of Wearable Sensors," Information Technology in Biomedicine, IEEE Transactions on, vol. 16, no. 3, pp. 478-487, 2012.

[13] G. Rigas, A. T. Tzallas, D. G. Tsalikakis, S. Konitsiotis, and D. I. Fotiadis, "Real-time quantification of resting tremor in the Parkinson's disease.," Conference Proceedings of the International Conference of IEEE Engineering in Medicine and Biology Society, vol. 2009, pp. 1306 1309, 2009.

[14] M. G. Tsipouras, A. T. Tzallas, G. Rigas, P. Bougia, D. I. Fotiadis, and S Konitsiotis, "Automated Levodopa-induced dyskinesia assessment," Engineering in Medicine and Biology Society EMBC 2010 Annual International Conference of the IEEE, vol. 2010, pp. 2411-2414, 2010.

[15] M. G. Tsipouras, A. T. Tzallas, D. I. Fotiadis, and S. Konitsiotis, "On automated assessment of Levodopa-induced dyskinesia in Parkinson's disease," 2011 Annual International Conference of the IEEE Engineering in Medicine and Biology Society, vol. 16, no. 1, pp. 58-61, 2001.

[16] J. Cancela, M. Pansera, M. Pastorino, L. Pastor, and M. Arredondo, "Automatic assessment of bradykinesia severity in patients with Parkinson's disease," in 7th International Conference on Wearable Micro and Nano Technologies for Personalized Health, 2010.

[17] M. Pastorino, J. Cancela, M. T. Arredondo, M. Pansera, L. Pastor-Sanz, F. Villagra, M. A. Pastor, and J. A. Martin, Assessment of bradykinesia in Parkinson's disease patients through a multi-parametric system. IEEE, 2011, pp. 1810-1813.

[18] J. Cancela, M. Pastorino, M. T. Arredondo, M. Pansera, L. Pastor-Sanz, F Villagra, M. A. Pastor, and A. P. Gonzalez, Gait assessment in Parkinson's disease patients through a network of wearable accelerometers in unsupervised environments. IEEE, 2011, pp. 2233 2236.

[19] S. Hignett and L. McAtamney, "Rapid entire body assessment (REBA).," Applied Ergonomics, vol. 31, no. 2, pp. 201-205, 2000. 